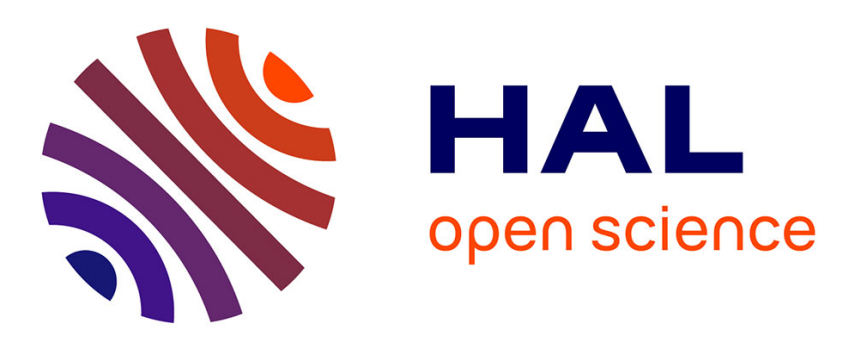

\title{
Mise au point d'un scintillateur gazeux à faible bruit de fond
}

D. Paya, J. Blons, J. Fermandjian, A. Fubini, Y. Pranal

\section{To cite this version:}

D. Paya, J. Blons, J. Fermandjian, A. Fubini, Y. Pranal. Mise au point d'un scintillateur gazeux à faible bruit de fond. Revue de Physique Appliquée, 1969, 4 (2), pp.289-290. 10.1051/rphysap:0196900402028901 . jpa-00243265

\section{HAL Id: jpa-00243265 https://hal.science/jpa-00243265}

Submitted on 1 Jan 1969

HAL is a multi-disciplinary open access archive for the deposit and dissemination of scientific research documents, whether they are published or not. The documents may come from teaching and research institutions in France or abroad, or from public or private research centers.
L'archive ouverte pluridisciplinaire HAL, est destinée au dépôt et à la diffusion de documents scientifiques de niveau recherche, publiés ou non, émanant des établissements d'enseignement et de recherche français ou étrangers, des laboratoires publics ou privés. 


\title{
MISE AU POINT D’UN SGINTILLATEUR GAZEUX A FAIBLE BRUIT DE FOND
}

\author{
D. PAYA, J. BLONS, J. FERMANDJIAN, A. FUBINI et Y. PRANAL, \\ Service des Mesures Neutroniques Fondamentales, C.E.N., Saclay.
}

Résumé. - On décrit un scintillateur gazeux de grandes dimensions dans lequel la mise en coïncidence de photomultiplicateurs a permis de réduire considérablement le bruit de fond le rendant particulièrement adapté à la mesure de sections efficaces de fission très faibles.

Abstract. - A large-volume gas scintillator is described in which, by means of coincidences between photomultipliers, it has been possible to significantly reduce the background. Such an arrangement is particularly suitable for the measurement of very small fission cross-sections.

I. Introduction. - La mesure des sections efficaces de fission au-dessous du seuil, par des neutrons de basse énergie, impose un certain nombre de conditions dans le choix du détecteur. Gelles-ci sont liées, d'une part à la valeur extrêmement faible de la section efficace, d'autre part à la radioactivité naturelle de l'échantillon étudié et de ses descendants. Ces conditions sont souvent contradictoires. C'est ainsi que le faible taux de comptage incite à augmenter la quantité d'échantillon alors que les empilements d'impulsions dues aux rayons $\alpha$, si on utilise un détecteur de fragments de fission, imposent rapidement une limite à cette augmentation. Le bruit de fond est aussi un facteur à considérer, car c'est de son importance que va dépendre la possibilité de déceler les résonances les plus petites. Le scintillateur gazeux a été choisi parce qu'il peut contenir une quantité assez grande d'élément fissile et qu'il donne des scintillations brèves qui présentent un risque d'empilement réduit. Un soin tout particulier a été pris pour diminuer le bruit de fond.

II. Description. - Le scintillateur est représenté schématiquement sur la figure 1 . Il est constitué de six cellules identiques contenues dans un corps cylin-

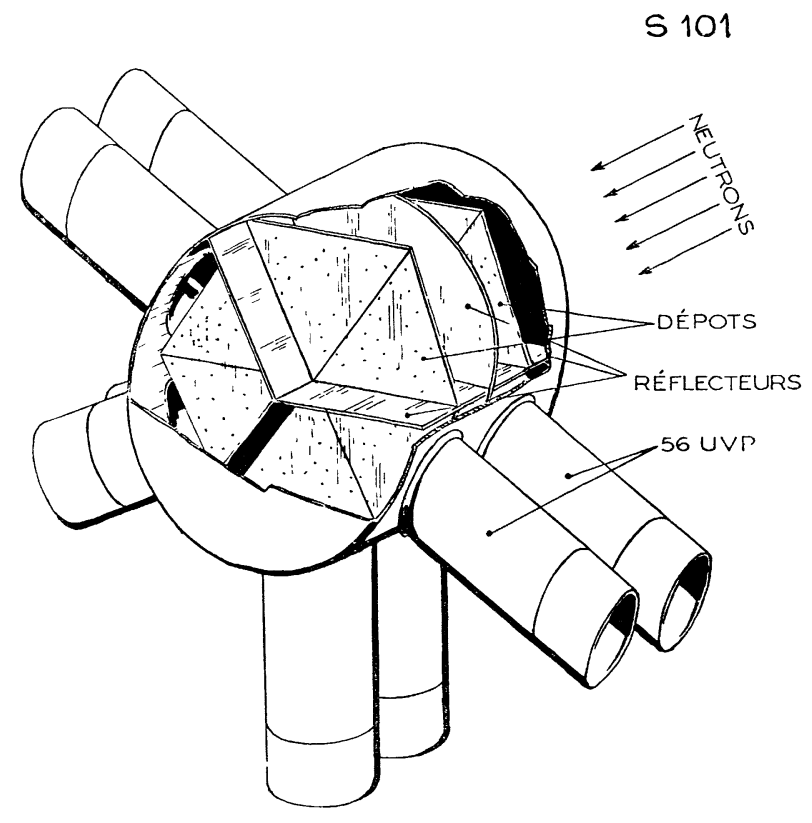

FIG. 1. - Vue du scintillateur gazeux. 
drique. Les parois des cellules sont des feuilles d'aluminium de qualité OAR (Oxydation Anodique Réfléchissante) qui possèdent un coefficient de réflexion très élevé. Chaque cellule contient deux dépôts triangulaires de ${ }^{237} \mathrm{~Np}$ sur support d'aluminium. Les dépôts sont obtenus par peintures et calcinations successives jusqu'à l'obtention d'une épaisseur de $2 \mathrm{mg} / \mathrm{cm}^{2}$ de neptunium sur chaque face du support. Ils sont ensuite recouverts d'une couche d'aluminium de $50 \mu \mathrm{g} / \mathrm{cm}^{2}$ obtenue par évaporation sous vide. Chaque cellule est couplée optiquement par l'intermédiaire de fenêtres de quartz, à deux photomultiplicateurs 56 UVP dont les axes sont dans le plan des dépôts. Le découpage en cellules du volume scintillant assure une bonne collection de lumière vers les photomultiplicateurs et limite leur champ de vision, ce qui réduit le risque d'empilement. La quantité totale de neptunium introduite dans le scintillateur est de $2 \mathrm{~g}$, ce qui représente une activité de $5 \times 10^{7}$ rayons $\alpha$ par seconde. Cependant, il serait possible de tolérer sans risque d'empilement trop important une activité deux à trois fois plus grande.

Le xénon a été choisi comme gaz scintillant parce qu'il donne des scintillations plus importantes et une meilleure discrimination entre rayons $\alpha$ et fragments de fission. L'utilisation de joints d'indium rend inutiles la circulation et la purification du gaz; plusieurs mois après le remplissage, aucune variation n'a été constatée dans la taille des impulsions.

III. Réduction du bruit de fond. - Le scintillateur a été utilisé dans le faisceau de neutrons pulsé fourni par l'accélérateur linéaire de $45 \mathrm{MeV}$ de Saclay, les énergies de neutrons étant mesurées par temps de vol [1-3]. Il est apparu alors deux composantes de bruit de fond relativement importantes compte tenu du faible taux de fission [1]. La première est due au xénon dont il est possible d'identifier les résonances; la seconde subsiste en l'absence de gaz scintillant et reproduit la forme du spectre des neutrons incidents. L'hypothèse la plus vraisemblable sur la cause de ce bruit de fond réside dans la détection, par les photomultiplicateurs eux-mêmes, de neutrons diffusés par le gaz ou les matériaux de structure. Il est difficile de réduire encore l'importance de ces derniers sans nuire à la solidité mécanique de l'ensemble. Cependant, la mise en coïncidence des photomultiplicateurs couplés à la même cellule supprime pratiquement les deux composantes et assure que le détecteur n'est plus sensible qu'aux scintillations émises au voisinage du centre de la cellule.

Le bruit de fond d'origine neutronique étant éliminé par le système de coïncidence, les seuils de discrimination peuvent être réglés à des valeurs plus basses. Il est alors possible de détecter des fragments qui, émis à des angles plus grands par rapport à la normale au dépôt, ont perdu une partie de leur pouvoir ionisant avant de pénétrer dans le gaz et donnent une scintillation de plus faible intensité. Cette plus grande efficacité de détection jointe au faible bruit de fond permet de mettre en évidence des résonances beaucoup plus petites.

Il reste à s'assurer que les résonances observées sont bien des résonances de fission. En effet, on sait que les scintillateurs gazeux peuvent être sensibles aux rayons $\gamma$. Or, la section efficace de capture radiative de ${ }^{237} \mathrm{~Np}$ est beaucoup plus grande que la section efficace de fission. On peut cependant constater que les résonances disparaissent effectivement si on recouvre les dépôts de feuilles d'aluminium d'épaisseur $20 \mu \mathrm{m}$, opaques aux fragments de fission, mais transparentes aux rayons $\gamma$.

IV. Conclusion. - La comparaison avec une chambre d'ionisation a montré que le scintillateur gazeux équipé du système de coïncidences que nous avons décrit ici possède, pour une efficacité comparable, un bruit de fond huit fois plus faible. Cet avantage, joint à la possibilité d'utiliser une plus grande quantité de matière fissile, en fait un détecteur bien adapté à la mesure de sections efficaces de fission de faible valeur comme celle des noyaux non fissiles par neutrons lents. Pour les noyaux fissiles, la section efficace entre résonances, qui est petite et souvent mal connue, joue un rôle important dans l'analyse par un formalisme à plusieurs niveaux. Dans ce domaine aussi, le scintillateur gazeux à faible bruit de fond pourrait trouver des applications très intéressantes.

Remerciements. - Les auteurs tiennent à remercier MM. Eggermann et Dumazert qui se sont chargés de la construction et du montage mécanique du scintillateur, ainsi que Mlle Valentin qui a réalisé les dépôts de neptunium.

\section{BIBLIOGRAPHIE}

[1] Paya (D.) et al., Nucl. Data for Reactors, I.A.E.A., Vienne, 1967, II, 128.

[2] Paya (D.) et al., J. Physique, 1968, 29, C1-159.

[3] Fubini (A.) et al., Phys. Rev. Letters, 1968, 20, 24, 1373. 